\title{
Rainy Garden
}

\author{
Annie Sungkajun \\ Soft Interaction Lab \\ Department of VIZ \\ Texas A\&M University \\ 3137 TAMU, USA \\ asungkajun@tamu.edu
}

\author{
Jinsil Hwaryoung Seo \\ Soft Interaction Lab \\ Department of VIZ \\ Texas A\&M University \\ 3137 TAMU, USA \\ hwaryoung@tamu.edu
}

\section{INTRODUCTION}

Rainy Garden is an interactive installation that creates a moment of sensory awakening and playfulness through tangible and immersive interactions. Comprised of seven umbrellas hung from the ceiling, the umbrellas contain an Arduino compatible circuitry that is embedded in each umbrella to create sensory immersion. This project was inspired by the sense of refuge that one gets from nature. Grasping a handle of each umbrella provides a personal boundary with distinct sensory stimulations and unique floral design print.

As an overview, the umbrellas resemble flowers laid out in a floating garden. UV LEDs attached to the outer surface of the umbrellas slowly animate, creating the effect of raindrops. Once a visitor holds a handle of the umbrella and stays underneath it, the space responds to the viewer and evokes narratives through haptic and either visual or auditory experiences. Depending on the quality of touch by a visitor, hidden visual patterns, vibrations, cricket-like sounds will appear and create a sensory-rich environment.

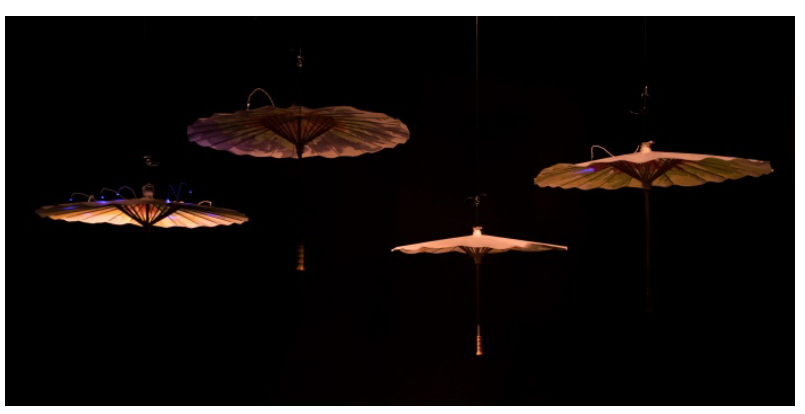

Figure 1: Rainy Garden installation.

\section{BACKGROUND}

Rainy Garden is inspired by the childhood memories one has about nature, be it jumping in the rain, gardening, the fresh smell of dew after rain, etc. Nature is a refuge that provides a sense of shelter and comfort. It is from this nostalgic inspiration that we gain the core aesthetic components of our work. Each umbrella within Rainy Garden becomes that same refuge of nature, an idealised immersion - immersion without vulnerability.

Understanding the intimate relationship between the human and nature/the world is further elaborated in Rainy Garden. Immersion can be considered as a phenomenologically interwoven state between environments/nature and the human, where the mental and physical merge like the seashore. From this art installation, we found that many participants' subjective experiences of this project shared similar qualities, including terms such as meditative, playful, natural, therapeutic, and poetic. Rainy Garden extends our artistic journey of exploring the concept of immersion focusing on experiential qualities.

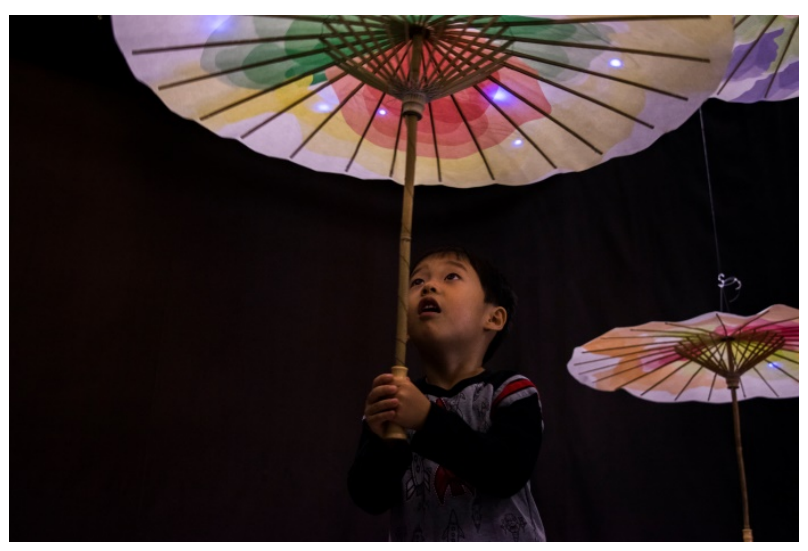

Figure 2: Child interacting with light-responsive umbrella.

\subsection{Interaction}

Each umbrella provides a different sensory experience: four umbrellas create visual and haptic immersion, while the other three evoke auditory and haptic immersion. The umbrellas have their own initial state and responds in relation to the 
quality of touch of the viewer. The visual and haptic umbrellas' initial state begins with the UV lights flickering at a fast rate to resemble raindrops falling atop the umbrella. When a viewer becomes close and grasps the handle of the umbrella, the lights will pulse at a slower rate so that the viewer will be allowed to see the subtle patterns on the umbrella. For the auditory and haptic umbrellas, two small buzzers play cricket-like sounds in their initial setting. Once the viewer touches the handle, the viewer will be able to feel soft vibrations generating in small bursts reverberating through the stick, to simulate the feeling of raindrops falling upon skin.

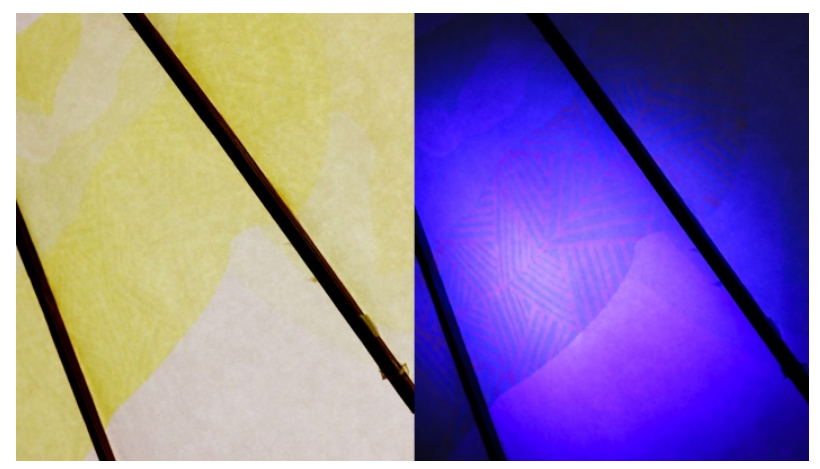

Figure 3: Close-up of Rainy Garden umbrella (left), Close-up of the area shown under UV light revealing hidden patterns (right)

\subsection{Implementation}

The seven umbrellas are hung from the ceiling, using nylon fishing wires that are strong enough to hold over $50 \mathrm{lbs}$. Unique printed flower patterns are then glued on each wooden umbrella structure and leaf-like patterns are hand drawn on the print, using UV markers. The ink is hidden and can be only seen under UV light. Both types of umbrellas are equipped with a Light BlueBean+ board and a capacitive sensor circuit. Light BlueBean+ is an Arduino-compatible board that can be programmed wirelessly using Bluetooth Low Energy and has a $600 \mathrm{mAh}$ rechargeable battery. The visual and haptic umbrellas utilise eight UV LEDs and one Lilypad vibe board connected to a Light BlueBean+ board atop the umbrella's wooden structure. For the auditory and haptic umbrellas, two Lilypad buzzer boards and one Lilypad vibe board are connected similarly as the visual and haptic umbrellas.

\section{CONCLUSION}

Rainy Garden explores the concept of feeling immersed in nature, by using umbrellas with distinct sensory simulations. With their own type of interactions, the umbrellas can evoke different responses from the viewer, as each person recalls their own experience with nature. The installation can be expanded to additional umbrellas, of varying degrees in height, with a combination of sensory experiences that viewers can explore through, much like an actual garden. 